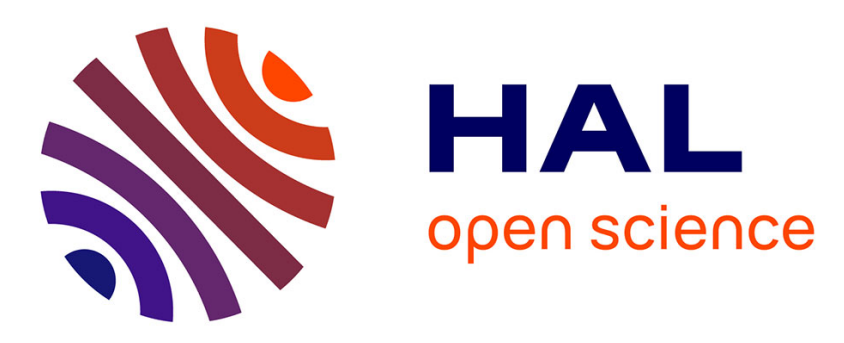

\title{
ECG denoising and fiducial point extraction using an extended Kalman filtering framework with linear and nonlinear phase observations
}

Mahsa Akhbari, Mohammad-Bagher Shamsollahi, Christian Jutten, Antonis Armoundas, Omid Sayadi

\section{To cite this version:}

Mahsa Akhbari, Mohammad-Bagher Shamsollahi, Christian Jutten, Antonis Armoundas, Omid Sayadi. ECG denoising and fiducial point extraction using an extended Kalman filtering framework with linear and nonlinear phase observations. Physiological Measurement, 2016, 37 (2), pp.203. 10.1088/0967-3334/37/2/203 . hal-01263915

\section{HAL Id: hal-01263915 \\ https://hal.science/hal-01263915}

Submitted on 28 Jan 2016

HAL is a multi-disciplinary open access archive for the deposit and dissemination of scientific research documents, whether they are published or not. The documents may come from teaching and research institutions in France or abroad, or from public or private research centers.
L'archive ouverte pluridisciplinaire HAL, est destinée au dépôt et à la diffusion de documents scientifiques de niveau recherche, publiés ou non, émanant des établissements d'enseignement et de recherche français ou étrangers, des laboratoires publics ou privés. 


\title{
ECG denoising and fiducial point extraction using an extended Kalman filtering framework with linear and nonlinear phase observations
}

\author{
Mahsa Akhbari ${ }^{1,2}$, Mohammad B. Shamsollahi ${ }^{1}$, \\ Christian Jutten ${ }^{2}$, Antonis A. Armoundas ${ }^{3}$ and \\ Omid Sayadi ${ }^{3}$ \\ 1 Biomedical Signal and Image Processing Laboratory (BiSIPL), Department of \\ Electrical Engineering, Sharif University of Technology, Tehran, Iran \\ 2 GIPSA-Lab, Department of Images and Signals, CNRS and University of \\ Grenoble-Alpes, France \\ 3 Cardiovascular Research Center, Massachusetts General Hospital, Harvard \\ Medical School, Charlestown, MA 02129 \\ E-mail: mahsa.akhbari@gipsa-lab.grenoble-inp.fr, mbshams@sharif.edu, \\ christian.jutten@gipsa-lab.grenoble-inp.fr, aarmoundas@partners.org \\ and sayadi.omid@mgh.harvard.edu
}

\begin{abstract}
In this paper we propose an efficient method for denoising and extracting fiducial point (FP) of ECG signals. The method is based on a nonlinear dynamic model which uses Gaussian functions to model ECG waveforms. For estimating the model parameters, we use an extended Kalman filter (EKF). In this framework called EKF25, all the parameters of Gaussian functions as well as the ECG waveforms (P-wave, QRS complex and T-wave) in the ECG dynamical model, are considered as state variables. In this paper, the dynamic time warping method is used to estimate the nonlinear ECG phase observation. We compare this new approach with linear phase observation models. Using linear and nonlinear EKF25 for ECG denoising and nonlinear EKF25 for fiducial point extraction and ECG interval analysis are the main contributions of this paper. Performance comparison with other EKF-based techniques shows that the proposed method results in higher output SNR with an average SNR improvement of $12 \mathrm{~dB}$ for an input SNR of $-8 \mathrm{~dB}$. To evaluate the FP extraction performance, we compare the proposed method with a method based on partially collapsed Gibbs sampler and an established EKF-based method. The mean absolute error and the root mean square error of all FPs, across all databases are $14 \mathrm{msec}$ and $22 \mathrm{msec}$, respectively, for our proposed method, with an advantage when using a nonlinear phase observation. These errors are significantly smaller than errors obtained with other methods. For ECG interval analysis, with an absolute mean error and a root mean square error of about $22 \mathrm{msec}$ and $29 \mathrm{msec}$, the proposed method achieves better accuracy and smaller variability with respect to other methods.

Keywords: Electrocardiogram (ECG), Extended Kalman Filter (EKF), Dynamic Time Warping (DTW), Fiducial Point Extraction, Denoising.
\end{abstract}




\section{Introduction}

The electrocardiogram (ECG) serves as the most important non-invasive diagnostic tool for cardiologists. Fiducial point (FP) extraction of ECG signal refers to identifying the location of the peak as well as the onset and offset of the P-wave, QRS complex and T-wave which convey clinically useful information.

Up to now, many methods have been used to detect the QRS complex (Kohler et al 2002). These methods are based on derivative-based algorithms (Holsinger et al 1971), filtering approaches (digital filters (Yu et al 1985), adaptive filters (Soria et al 1998)), mathematical transformations (wavelet ( $\mathrm{Li} \mathrm{C}$ et al 1995, Martinez J P et al 2004, Dumont et al 2010), filter banks (Afonso et al 1999), phasor transform (Martinez A et al 2010)), classification methods (neural network approaches (Hu et al 1993), support vector machine (SVM) (Mehta et al 2008), fuzzy C-means algorithm (Mehta et al 2009)), hidden Markov models (HMM) (Coast et al 1990, Hughes et al 2004a, Hughes et al 2006. Andreao et al 2006a, Andreao et al 2006b), automated method (Christov et al 2007) and mathematical morphology methods (Sun et al 2005). Adaptive filters, wavelet transform, SVM, mathematical morphology methods, HMM and Partially Collapsed Gibbs Sampler (PCGS) (Lin et al 2010, Lin et al 2011a) have also been used for $\mathrm{P}$ - and $\mathrm{T}$-wave delineation.

However, under commonly met conditions, the ECG signal may be contaminated by the recording instrument, the bioelectric activity of the tissues not belonging to the area of diagnostic interest and motion artifact due to movement of the patient or the electrode (Ardhapurkaret al 2012). Such contamination may alter clinically important information of the signal, therefore ECG denoising is vital.

ECG denoising and fiducial point extraction have been used in many ECG applications. For example, Maheshwari et al. (2013) proposed an automated algorithm for online detection of the fragmented QRS complex and identification of its various morphologies. In this application, ECG denoising and feature extraction are essential preprocessing steps. Mazomenos et al. (2013) proposed a low-complexity ECG feature extraction algorithm for mobile healthcare applications. This method requires the initial estimation of the QRS complex, P- and T-waves, as well as their onset and offset. Bono et al. (2014) developed an automated updated "Selvester QRS scoring" system, which requires the onset and offset of the P-wave and QRS complex as the input to their model.

For that matter, a nonlinear dynamical model for generating synthetic ECG signals has been developed by McSharry et al. (2003). Prior work from our group has extended and modified the underlying model of the Kalman filter (process equations), as well as the corresponding series of ECG and phase measurements observed over time (observations). This model, parameters of which are estimeted by an extended Kalman filter (EKF), has been used in the following applications: ECG denoising (Sameni et al. (2007, 2008), Sayadi et al. (2008, 2010b), Akhbari et al. (2012)), ECG fiducial point extraction (Sayadi et al. (2009)) and Premature ventricular contraction (PVC) detection (Sayadi et al. (2010a)).

Since these EKF-based methods assume linear phase observation, when abnormal waveforms appear intermittently in some ECG cycles, they are unable to simultaneously filter the normal and abnormal ECG segments.

In this paper, we propose a novel efficient method for ECG denoising and FP extraction using nonlinear phase observation. In this framework called EKF25, all the parameters of Gaussian functions as well as the ECG waveforms (P-wave, QRS 
complex and T-wave) in the ECG dynamical model, are considered as state variables. To validate our method, we use signals from the MIT-BIH arrhythmia database (MIT BIH Arrhythmia Database), the physionet QT database (QT Database, Laguna et al 1997) and a swine ECG database (Sayadi et al 2014).

Limited parts of this paper have been published in two international conferences (Akhbari et al 2013a, Akhbari et al 2013b). This paper contains significant new contributions, specifically with regards to the methods and results based on nonlinear phase, and experimental results extended to 3 databases. We have already proposed the EKF25 with two observations (EKF25-2obs) (Akhbari et al 2013a) and EKF25 with four observations (EKF25-4obs) (Akhbari et al 2013b). In prior studies we considered a linear phase observation for EKF models and used them for FP extraction. In this paper, we explain the EKF25 models and systematically derive all EKF equations. Therefore, the original contributions of this paper include denoising based on linear and nonlinear EKF25 models as well as FP extraction and interval analysis based on nonlinear EKF25, described in subsections 3.2, 4.1, 5.1, 5.2 and 5.3.

The paper is organized as follows. Related work are described in section 2, in section 3 we explain our proposed method, and in section 4 we discuss three applications (ECG denoising, FP extraction and interval analysis). Section 5 presents the experimental results, and finally section 6 concludes the paper.

\section{Related Work}

\subsection{ECG Kalman Filtering Framework}

McSharry et al. (2003) have proposed a synthetic ECG generator, which is based on a nonlinear dynamic model. Sameni et al. (2007) transformed it into the polar coordinates from Cartesian coordinates and proposed an EKF-based framework (called "EKF2") which has two state variables and two observations. The discrete stateequations of this model are as follows:

$$
\left\{\begin{array}{l}
\varphi_{k+1}=\left(\varphi_{k}+\omega_{k} \delta\right) \bmod (2 \pi) \\
z_{k+1}=-\sum_{i} \delta \frac{\alpha_{i k} \omega_{k}}{b_{i k}^{2}} \Delta \theta_{i k} \exp \left(-\frac{\Delta \theta_{i k}^{2}}{2 b_{i k}^{2}}\right)+z_{k}+\eta_{k}
\end{array}\right.
$$

where $\varphi_{k}$ is the phase of ECG and $\omega_{k}$ is the beat-to-beat angular frequency of the RR interval. In this model $z_{k}$ is a state variable which is a sum of 5 Gaussian functions $(i \in\{P, Q, R, S, T\})$. Each Gaussian function is defined with three main parameters: $\alpha_{i k}, b_{i k}$ and $\theta_{i k}$, which correspond to the amplitude, width and location of the Gaussian functions and $\Delta \theta_{i k}=\left(\varphi_{k}-\theta_{i k}\right) \bmod (2 \pi) ; \delta$ is the sampling period, $\eta_{k}$ is a random additive noise that models the inaccuracies of the dynamic model. System state and process noise vectors are defined as:

$$
\begin{aligned}
& \underline{x}_{k}=\left[\varphi_{k}, z_{k}\right]^{T} \\
& \underline{w}_{k}=\left[\alpha_{P_{k}}, \ldots, \alpha_{T_{k}}, b_{P_{k}}, \ldots, b_{T_{k}}, \theta_{P_{k}}, \ldots, \theta_{T_{k}}, \omega_{k}, \eta_{k}\right]^{T}
\end{aligned}
$$

In this model, the noisy recording, $s_{k}$, (see (3)) refers to the ECG observation (second observation) of the EKF, while the phase (first observation) is denoted as $\Phi_{k}$. Then, by definition, the R-wave peak is always assumed to be located at $\varphi_{k}=0$ and the ECG content lying between two consecutive $\mathrm{R}$-wave peaks is assigned a linear phase between 0 and $2 \pi$ (or $-\pi$ and $\pi$ ). By detecting the R-wave peaks, the linearly approximated 
phase is obtained and considered as the phase observation, $\Phi_{k}$ (Sameni et al 2007). Therefore, the observation equations are as follows:

$$
\left\{\begin{array}{c}
\Phi_{k}=\varphi_{k}+v_{1 k} \\
s_{k}=z_{k}+v_{2 k}
\end{array}\right.
$$

where $\underline{v}_{k}=\left[v_{1 k}, v_{2 k}\right]^{T}$ is the observation noise vector.

Sayadi et al. extended the EKF2 framework and added the 3 parameters of each of the 5 Gaussian functions in (1), as states to EKF2, and called this extended model "EKF17". This approach was successfully used for ECG denoising, compression (Sayadi et al 2008) and beat segmentation of normal ECG signals (Sayadi et al 2009). The system state and process noise vectors of this model are defined as:

$$
\begin{aligned}
& \underline{x}_{k}=\left[\varphi_{k}, z_{k}, \alpha_{P_{k}}, \ldots, \alpha_{T_{k}}, b_{P_{k}}, \ldots, b_{T_{k}}, \theta_{P_{k}}, \ldots, \theta_{T_{k}}\right]^{T} \\
& \underline{w}_{k}=\left[\omega_{k}, \eta_{k}, u_{1, k}, \ldots, u_{15, k}\right]^{T}
\end{aligned}
$$

Sayadi et al. also described a Gaussian wave-based state space model whose characteristic waveforms, i.e. P-wave, QRS complex and T-wave are considered as state variables. This model, with 4 states, was called "EKF4" and was used for ECG denoising (Sayadi et al 2010b) and PVC detection (Sayadi et al 2010a).

To account for heart rate variability, Akhbari et al. (2012) introduced a firstorder autoregressive (AR) model for angular velocity of ECG $\left(\omega_{k}\right)$, in the dynamical state-space model, leading to an EKF model with 3 state equations, denoted EKF3, used for ECG denoising.

\subsection{Partially Collapsed Gibbs Sampler Method (PCGS)}

Lin et al. (2010) introduced a novel hierarchical Bayesian model that simultaneously estimates the $\mathrm{P}$ - and T-wave delineations. This model takes into account a prior distribution of the unknown parameters (such as the wave locations and amplitude, and waveform coefficients). These prior distributions are combined with the likelihood of the observed data to provide the posterior distribution of the unknown parameters. The posterior distribution depends on hyper-parameters that can be fixed a priori or estimated from the observed data (Lin et al 2010). This method is based on a partially collapsed Gibbs sampler (PCGS) which can estimate the onset, peak position and offset of P- and T-waves.

\section{Proposed Methods}

\subsection{EKF25 approach with linear phase observation}

Using the EKF4 and EKF17 methods and assuming small changes of the P-wave, QRS complex and T-wave morphology during consecutive cardiac cycles, we introduce a first-order AR model for each of the Gaussian parameters describing an ECG waveform, and also consider three separate states for ECG waveforms (P-wave, QRS complex and T-wave). Discrete state and observation equations of this model (EKF25 with two observations) are defined in (5) and (6), respectively. Here we use "C" to denote the QRS complex. 


$$
\begin{aligned}
& \left\{\begin{array}{l}
\varphi_{k+1}=\left(\varphi_{k}+\omega_{k} \delta\right) \bmod (2 \pi) \\
P_{k+1}=-\sum_{i \in\left\{P_{1}, P_{2}\right\}}^{\operatorname{mos}} \delta \frac{\alpha_{i k} \omega_{k}}{b_{i k}^{2}} \Delta \theta_{i k} \exp \left(-\frac{\Delta \theta_{i k}^{2}}{2 b_{i k}^{2}}\right)+P_{k}+\eta_{P_{k}} \\
C_{k+1}=-\sum_{i \in\{Q, R, S\}}^{i} \delta \frac{\alpha_{i k} \omega_{k}}{b_{i k}^{2}} \Delta \theta_{i k} \exp \left(-\frac{\Delta \theta_{i k}^{2}}{2 b_{i k}^{2}}\right)+C_{k}+\eta_{C_{k}} \\
T_{k+1}=-\sum_{i \in\left\{T_{1}, T_{2}\right\}} \delta \frac{\alpha_{i k} \omega_{k}}{b_{i k}^{2}} \Delta \theta_{i k} \exp \left(-\frac{\Delta \theta_{i k}^{2}}{2 b_{i k}^{2}}\right)+T_{k}+\eta_{T_{k}} \\
\alpha_{i, k+1}=\alpha_{i, k}+u_{j, k}, j=\{1, \cdots, 7\} \\
b_{i, k+1}=b_{i, k}+u_{j, k}, j=\{8, \cdots, 14\} \\
\theta_{i, k+1}=\theta_{i, k}+u_{j, k}, j=\{15, \cdots, 21\} \\
i \in\left\{P_{1}, P_{2}, Q, R, S, T_{1}, T_{2}\right\}
\end{array}\right. \\
& \left\{\begin{array}{l}
\Phi_{k}=\varphi_{k}+v_{1 k} \\
z_{k}=P_{k}+C_{k}+T_{k}+v_{2 k}
\end{array}\right.
\end{aligned}
$$

In (5), the first state is the phase of the ECG. The second, third and fourth ones are distinct ECG waveforms (P-wave, QRS complex and T-wave) which are separately considered as states. The parameters of the Gaussian functions are considered as the $5^{t h}$ to $25^{t h}$ state variables with first order AR dynamics but without corresponding observations. The system state and process noise vectors are defined as:

$$
\begin{aligned}
& \underline{x}_{k}=\left[\varphi_{k}, P_{k}, C_{k}, T_{k}, \alpha_{P_{1}, k}, \ldots, b_{P_{1}, k}, \ldots, \theta_{P_{1}, k}, \ldots, \theta_{T_{2}, k}\right]^{T} \\
& \underline{w}_{k}=\left[\omega_{k}, \eta_{P_{k}}, \eta_{C_{k}}, \eta_{T_{k}}, u_{1, k}, \ldots, u_{21, k}\right]^{T}
\end{aligned}
$$

In (6), the first observation is the linearly approximated phase of ECG, and the ECG observation $\left(z_{k}\right)$ is the sum of $P_{k}, C_{k}$ and $T_{k}$ states. Taking the sum of the estimated states provides an enhanced estimation of the overal cardiac beat. However, individual estimated ECG states (P-wave, QRS complex and T-wave) may have rising and falling trends (drifts, which are compensated by the summation) resulting from the inaccuracies in modeling dynamic baseline changes with a unique observation $z_{k}$, sum of $P_{k}, C_{k}$ and $T_{k}$ (for more information, the reader can refer to (Akhbari et al 2013b) specially Figs.1 and 4). Observation and measurement noise vectors are defined respectively as: $y_{k}=\left[\Phi_{k}, z_{k}\right]^{T}$ and $\underline{v}_{k}=\left[v_{1 k}, v_{2 k}\right]^{T}$.

To control the above mentioned rising and falling trends, we modify a previously described model, in which we consider four observations and call it "EKF25-4obs". The 25 discrete state equations of this model are the same as (5) and its 4 observation equations are:

$$
\left\{\begin{array}{l}
\Phi_{k}=\varphi_{k}+v_{1 k} \\
P P_{k}=P_{k}+v_{2 k} \\
C C_{k}=C_{k}+v_{3 k} \\
T T_{k}=T_{k}+v_{4 k}
\end{array}\right.
$$

In (8), the first equation corresponds to the phase observation and the others correspond to the P-wave, QRS complex and T-wave, respectively. In this case, the observation and measurement noise vectors are defined respectively as: $\underline{y}_{k}=$ $\left[\Phi_{k}, P P_{k}, C C_{k}, T T_{k}\right]^{T}$ and $\underline{v}_{k}=\left[v_{1 k}, v_{2 k}, v_{3 k}, v_{4 k}\right]^{T}$.

Thereafter, we determine three windows to segment the original ECG signal, and obtain the $P P_{k}, C C_{k}$ and $T T_{k}$ observations. Here the windows are defined as the 
difference between two sigmoid functions and have tunable rising and falling edges. Fig.1 shows these windows for the $\mathrm{P}$-wave, QRS complex and T-wave. In general, the beginning and end of these windows are defined such that the P-wave, QRS complex and T-wave correspond to ECG phase in interval $[-\pi, a],[a, b]$ and $[b, \pi]$, respectively. Following the polargram partitioning, previously described in (Sayadi et al 2010a), for normal beats we can assume that $a=-\pi / 6$ and $b=\pi / 6$. For signals with premature or delayed waves, the value of $a$ and $b$ parameters changes moderately and can be set as a fixed value, as: $-0.4 \pi \leq a \leq-\pi / 6$ and $\pi / 6 \leq b \leq 0.4 \pi$. The values of "a" and "b" are defined experimentally from the first beat of each record. These windows are defined in (9) and their shape is controlled with $\gamma$, set here to $\gamma=30$ (this value is determined experimentally and is fixed for all beats). Observations $P P_{k}, C C_{k}$ and $T T_{k}$ in (8) are calculated by multiplying the original (observed) ECG signal by the windows defined in (9). Fig.2 shows the original ECG and the observations $P P_{k}, C C_{k}$ and $T T_{k}$ for a typical ECG signal. In fact, $P P_{k}, C C_{k}$ and $T T_{k}$ are exactly overlapped on original ECG but here for better vision, we plot them with an offset.

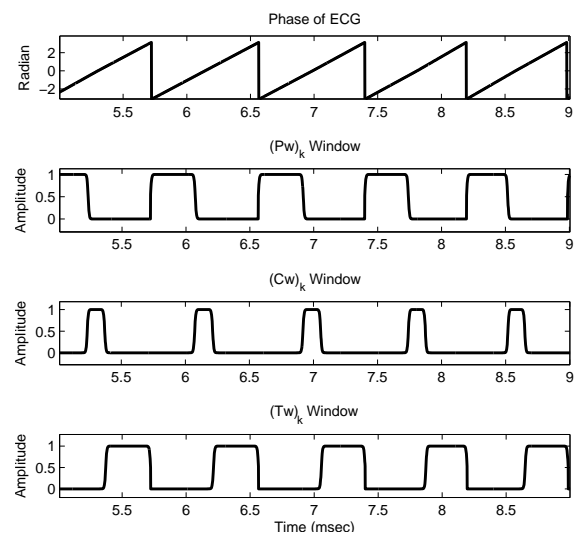

Figure 1. ECG phase and windows defined for P-wave, QRS complex and Twave.

$$
\begin{aligned}
(P w)_{k} & =\frac{1}{1+\exp ^{-\gamma\left(\Phi_{k}-(-\pi)\right)}}-\frac{1}{1+\exp ^{-\gamma\left(\Phi_{k}-(a)\right)}} \\
(C w)_{k} & =\frac{1}{1+\exp ^{-\gamma\left(\Phi_{k}-(a)\right)}}-\frac{1}{1+\exp ^{-\gamma\left(\Phi_{k}-(b)\right)}} \\
(T w)_{k} & =\frac{1}{1+\exp ^{-\gamma\left(\Phi_{k}-(b)\right)}}-\frac{1}{1+\exp ^{-\gamma\left(\Phi_{k}-(\pi)\right)}}
\end{aligned}
$$

Finally, to estimate the initial values for the state vector, the covariance matrix of each process and the measurement noise, we use the same procedure described by Sameni et al. (2007) and Sayadi et al. (2008) for the initialization of the modified EKF structure.

Here, process and measurement noise vectors are assumed to be independent. It should be noted that the algorithm is sensitive to the choice of these initial values. A robust selection strategy for the initial values of the two Gaussian functions describing the $\mathrm{P}$ and $\mathrm{T}$ waveforms would be to insure the final representation $\left(P_{1}+P_{2}\right.$ and $\left.T_{1}+T_{2}\right)$ can accurately model the asymmetric $\mathrm{P}$ and $\mathrm{T}$ waveforms. More details on convergence and stability of the EKF algorithms are discussed in (Sameni et al 2007) 


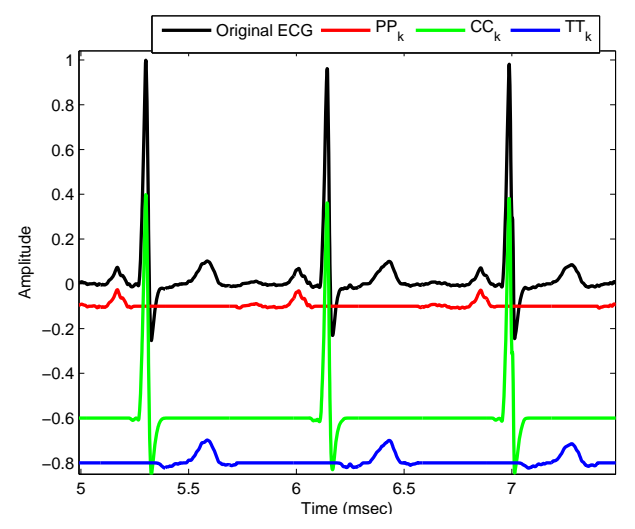

Figure 2. Original ECG and the observations $P P_{k}, C C_{k}$ and $T T_{k}$ for a typical ECG signal.

\subsection{EKF25 approach with nonlinear phase observation}

In prior works, the linearly approximated phase has been considered as a phase observation and this assumption is the same as having constant angular velocity $\left(\omega_{k}\right)$ during each beat, whereas in (1) and (5) and most of the previous studies, $\omega_{k}$ is considered as a process noise in EKF models. In other studies ((Akhbari et al 2012) and (Lin et al 2011b)) a first-order AR model is introduced for $\omega_{k}$ and is considered as a state of the model.

However, the linear phase observation is not accurate, especially for signals with major RR-interval deviations or signals with frequent abnormal beats that appear intermittently in the ECG. For constructing a more accurate approximation of the ECG phase observation, Niknazar et al. (2012) used the dynamic time warping (DTW) method. The DTW method measures the similarity between two sequences, which may vary in time or speed, to obtain an optimal match between two given sequences with certain restrictions (Myers et al 1981, Li Q et al 2012). For example, Zifan et al. (2006) used the piecewise derivative dynamic time warping (PDDTW) method for automated ECG segmentation, while Raghavendra et al. (2011) used DTW for arrhythmia detection in e-Healthcare systems.

The DTW distance between two sequences is computed as follows (Raghavendra et al 2011). Let the two sequences be represented as $X=\left(x_{1}, x_{2}, \cdots, x_{m}\right)$ of length $m$, and $Y=\left(y_{1}, y_{2}, \cdots, y_{n}\right)$ of length $n$. The DTW distance $D(X, Y)$ between $X$ and $Y$ is defined as $D(X, Y)=f(m, n)$, where:

$$
\begin{aligned}
& f(i, j)=\left\|x_{i}-y_{j}\right\|+\min \{f(i, j-1), f(i-1, j), f(i-1, j-1)\} \\
& \text { with } f(0,0)=0, f(i, 0)=f(0, j)=\infty \text { for } i=1,2, \cdots, m, j=1,2, \cdots, n .
\end{aligned}
$$

The notation $\left\|x_{i}-y_{j}\right\|$ represents Euclidean distance between two numerical values.

In this paper we propose using the DTW method to obtain a nonlinear phase observation of the ECG, which will be later used in the previously developed EKF2 and EKF25 models. The proposed DTW ensures that in the EKF2 and EKF25 models described by equations (3), (6) and (8), $\Phi_{k}$ has a nonlinear phase observation.

In our application, first a reference ECG beat is selected and a linear phase is assigned to it, then the current ECG beat and the reference ECG beat are nonlinearly warped to optimize their similarity of their nonlinear variations and an optimal curve 
is obtained. Finally, the nonlinear phase observation of the current ECG beat is obtained by aligning its linear phase on the linear phase of the reference ECG beat, according to the optimal curve. It is worth to mention that in DTW, the reference beat can be a typical or the average ECG beat. In both linear and nonlinear EKF models, the state and observation equations $((1),(3),(5),(6)$ and (8)) are the same and the only difference is that in nonlinear EKF, the nonlinear phase which is obtained by DTW is used as a phase observation $\left(\Phi_{k}\right)$ in (3), (6) and (8). Fig.3 shows the ECG signal with two PVCs and its defined linear and nonlinear phase observations.
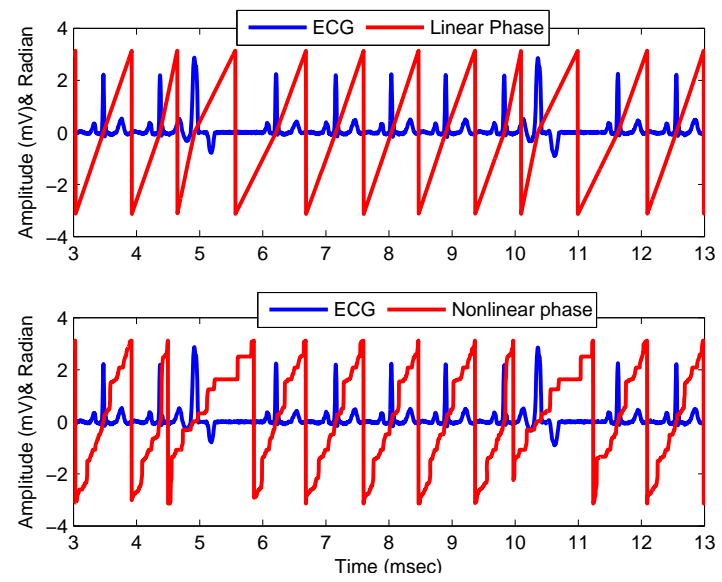

Figure 3. ECG signal and linear (top) and nonlinear (bottom) phase observation.

\section{Applications}

We sought to validate the proposed methods in three following applications: (i) ECG denoising, (ii) ECG fiducial point extraction, and (iii) ECG interval analysis. Table 1 shows the methods which are compared for each application.

Table 1. The methods compared in different applications.

\begin{tabular}{|l|c|c|c|}
\hline & \multicolumn{3}{|c|}{ Applications } \\
\hline Methods & ECG Denoising & ECG FP Extraction & ECG Interval Analysis \\
\hline Linear and Nonlinear EKF2 & YES & NO & NO \\
\hline Linear and Nonlinear EKF25-2obs & YES & NO & NO \\
\hline Linear and Nonlinear EKF25-4obs & YES & YES & YES \\
\hline EKF17 & NO & YES & YES \\
\hline PCGS & NO & YES & YES \\
\hline
\end{tabular}

\subsection{ECG denoising}

Sameni et al. (2007) used the EKF2 method (discussed in 2.1) for ECG denoising assuming a linear phase observation for the ECG. Given the superiority of EKF2 for ECG denoising in comparison with other benchmark methods (Sameni et al 2007), we will compare our proposed method only with EKF2. As described above, we first 


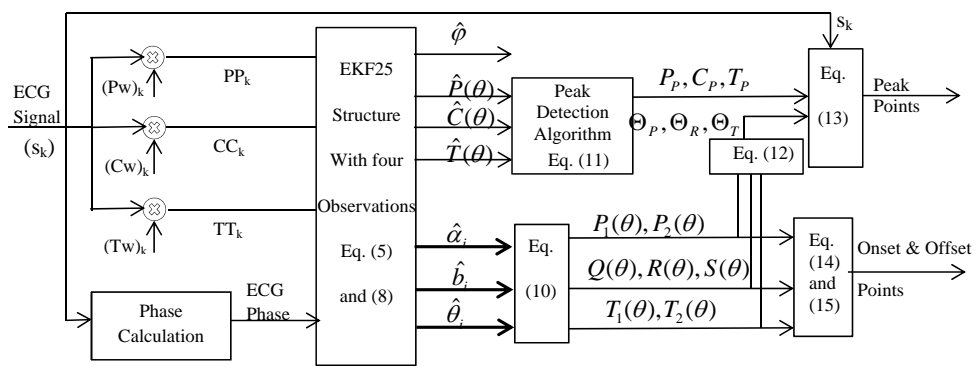

Figure 4. Blockdiagram of proposed EKF25 approach for FP extraction.

obtain a nonlinear phase observation for the ECG signal using the DTW technique, and then use the EKF2 method (Eqs. (1) and (3)), the EKF25 method with 2 observations (Eqs. (5) and (6)) and the EKF25 method with 4 observations (Eqs. (5) and (8)) for ECG denoising. In section 5.1, we compare the results of these three models. In EKF2, the second estimated state $\left(\hat{z}_{k}\right)$ and in EKF25, the summation of second, third and fourth estimated states $\left(\hat{P}_{k}+\hat{C}_{k}+\hat{T}_{k}\right)$ are considered as the denoised ECG.

\subsection{ECG fiducial point extraction}

For ECG FP extraction, we only use the EKF25 structure with four observations, because this model has no rising and falling trends, as previously discussed. Results of this application are presented in section 5.2. Fig.4 shows the blockdiagram of our proposed approach for finding the peak, onset and offset of ECG waveforms.

As presented above, we use the McSharry model which assumes that each ECG beat is a combination of $\mathrm{N}$ Gaussian functions (here $N=7$ ) and each Gaussian function is defined by 3 parameters $\alpha_{i}, b_{i}$ and $\theta_{i}$ (amplitude, width and location, respectively). First, all states of the model are estimated by EKF25; then we use the estimated Gaussian parameters (the $5^{\text {th }}$ to $25^{\text {th }}$ state variables) and construct the $P_{1}(\theta), P_{2}(\theta), Q(\theta), R(\theta), S(\theta), T_{1}(\theta)$ and $T_{2}(\theta)$ Gaussian functions as:

$$
i(\theta)=\hat{\alpha}_{i} \exp \left(-\frac{\left(\theta-\hat{\theta}_{i}\right)^{2}}{2 \hat{b}_{i}^{2}}\right), i \in\left\{P_{1}, P_{2}, Q, R, S, T_{1}, T_{2}\right\}
$$

In Fig.4, $\hat{P}(\theta), \hat{C}(\theta)$ and $\hat{T}(\theta)$ are the second, third and fourth estimated states by EKF 25, respectively and the $P_{1}(\theta), P_{2}(\theta), Q(\theta), R(\theta), S(\theta), T_{1}(\theta)$ and $T_{2}(\theta)$ functions are constructed from the estimated Gaussian parameters using (10). The proposed method for finding the peak position of ECG waveforms, consists of 3 steps:

(i) Based on the estimated ECG waveforms $(\hat{P}(\theta), \hat{C}(\theta)$ and $\hat{T}(\theta))$, find the location of the maximum absolute value of these waveforms by Eq. (11); these points are called $P_{P}, C_{P}$ and $T_{P}$ and are the preliminary estimations of the final peaks of the ECG waveforms.

$$
\begin{aligned}
& P_{P}=\underset{\theta}{\operatorname{argmax}}|\hat{P}(\theta)| \\
& C_{P}=\underset{\theta}{\operatorname{argmax}}|\hat{C}(\theta)| \\
& T_{P}=\underset{\theta}{\operatorname{argmax}}|\hat{T}(\theta)|
\end{aligned}
$$


(ii) Compute $P_{1}(\theta)+P_{2}(\theta), Q(\theta)+R(\theta)+S(\theta)$ and $T_{1}(\theta)+T_{2}(\theta)$ functions and find the location of the maximum absolute value of these functions by Eq. (12). These points are called $\Theta_{P}, \Theta_{R}$ and $\Theta_{T}$ and are the second candidate group for final peaks of the ECG waveforms.

$$
\begin{aligned}
& \Theta_{P}=\underset{\theta}{\operatorname{argmax}}\left|P_{1}(\theta)+P_{2}(\theta)\right| \\
& \Theta_{R}=\underset{\theta}{\operatorname{argmax}}|Q(\theta)+R(\theta)+S(\theta)| \\
& \Theta_{T}=\underset{\theta}{\operatorname{argmax}}\left|T_{1}(\theta)+T_{2}(\theta)\right|
\end{aligned}
$$

(iii) Use the decision rule (13), for computing the final peaks of ECG waveforms ( $P_{\text {peak }}$, $R_{\text {peak }}$ and $T_{\text {peak }}$ ), where $s_{k}$ is the observed (original) ECG signal. In (13), one compares the results obtained by (11) and (12) and chooses the maximum, as the final peak candidate.

$$
\begin{aligned}
& P_{\text {peak }}=\underset{\Theta_{P}, P_{P}}{\operatorname{argmax}}\left(\left|s_{k}\left(\Theta_{P}\right)\right|,\left|s_{k}\left(P_{P}\right)\right|\right) \\
& R_{\text {peak }}=\underset{\Theta_{R}, C_{P}}{\operatorname{argmax}}\left(\left|s_{k}\left(\Theta_{R}\right)\right|,\left|s_{k}\left(C_{P}\right)\right|\right) \\
& T_{\text {peak }}=\underset{\Theta_{T}, T_{P}}{\operatorname{argmax}}\left(\left|s_{k}\left(\Theta_{T}\right)\right|,\left|s_{k}\left(T_{P}\right)\right|\right)
\end{aligned}
$$

It is worth to mention that the peak of R-wave is considered as the peak of QRS complex and for all waves, maximum absolute amplitude is considered as the peak of waves to find the positive or negative peaks.

For the onset and offset points, if we model each of the P- and T-waves by one Gaussian function, we can assume that when any of the Gaussian functions representing $\mathrm{P}$ - or $\mathrm{T}$-waves in the dynamical model extends beyond 3 times its standard deviation, it reflects the onset or offset points, as described in (Sayadi et al 2009).

In this paper, we model each of the $\mathrm{P}$ and $\mathrm{T}$ waves with two Gaussian functions. Of course, $P_{1}(\theta)+P_{2}(\theta)$ and $T_{1}(\theta)+T_{2}(\theta)$ are no longer Gaussian functions and we cannot use the 3 -standard deviation threshold. Thus, we propose another method for finding the onset and offset of these waveforms. We introduce a suitable confidence bound $(\epsilon)$ for the termination of the constructed $P_{1}(\theta)+P_{2}(\theta), Q(\theta), S(\theta)$ and $T_{1}(\theta)+T_{2}(\theta)$ Gaussian functions, to determine the onset and offset of the corresponding waveform. We will discuss the significance of the values of $\epsilon$ in section 5.2.

The onset and offset of the QRS complex as well as P- and T-waves are determined by (14) and (15). First, we compute analytically the whole area under each constructed Gaussian function (with trapezoidal numerical integration):

$$
\begin{aligned}
& A_{P}=\int_{-\infty}^{\infty}\left|P_{1}(\theta)+P_{2}(\theta)\right| d \theta \\
& A_{Q}=\int_{-\infty}^{\infty}|Q(\theta)| d \theta \\
& A_{S}=\int_{-\infty}^{\infty}|S(\theta)| d \theta \\
& A_{T}=\int_{-\infty}^{\infty}\left|T_{1}(\theta)+T_{2}(\theta)\right| d \theta
\end{aligned}
$$


Then, we numerically determine:

$$
\begin{aligned}
& P_{o n}\left|\int_{-\infty}^{P_{o n}}\right| P_{1}(\theta)+P_{2}(\theta) \mid d \theta=\epsilon A_{P} \\
& P_{o f f}\left|\int_{P_{o f f}}^{\infty}\right| P_{1}(\theta)+P_{2}(\theta) \mid d \theta=\epsilon A_{P} \\
& Q R S_{o n}\left|\int_{-Q R S_{o n}}^{\infty}\right| Q(\theta) \mid d \theta=\epsilon A_{Q} \\
& Q R S_{o f f}\left|\int_{Q R S_{o f f}}^{\infty}\right| S(\theta) \mid d \theta=\epsilon A_{S} \\
& T_{o n}\left|\int_{-\infty}^{T_{o n}}\right| T_{1}(\theta)+T_{2}(\theta) \mid d \theta=\epsilon A_{T} \\
& T_{o f f}\left|\int_{T_{o f f}}^{\infty}\right| T_{1}(\theta)+T_{2}(\theta) \mid d \theta=\epsilon A_{T}
\end{aligned}
$$

To determine a suitable value for $\epsilon$, since we model each $\mathrm{P}$ and $\mathrm{T}$ wave by a sum of two Gaussian functions, we cannot use $\theta_{i} \pm 3 b_{i}$ to find the waveform onset and offset, as it is done in EKF17. Because the constructed $P_{1}(\theta)+P_{2}(\theta)$ and $T_{1}(\theta)+T_{2}(\theta)$ are not exactly Gaussian functions, we consider the total area under the constructed $P_{1}(\theta)+P_{2}(\theta)$ and $T_{1}(\theta)+T_{2}(\theta)$, between the waveform onset and offset is $99 \%$ of the total area under the whole constructed waveform. That means:

$$
\begin{aligned}
& \int_{P_{o f f}}^{P_{o f f}}\left|P_{1}(\theta)+P_{2}(\theta)\right| d \theta=0.99 A_{P} \\
& \int_{T_{o n}}^{T_{o f f}}\left|T_{1}(\theta)+T_{2}(\theta)\right| d \theta=0.99 A_{T}
\end{aligned}
$$

where, $A_{P}$ and $A_{T}$ are the total area under curve for the P- and T-waves, respectively. So the $\epsilon$ value in $(15)$ can be defined as: $\epsilon=(1-0.99) / 2=0.005$ for all waveforms. We consider a unique $\epsilon$ value to find waveform onset and offset of all signals.

\subsection{ECG Interval Analysis}

Having estimated the FPs, we sought to assess the performance of the proposed methods in measuring ECG intervals. As the PCGS method only estimates the peak, onset and offset of $\mathrm{P}$ - and T-waves, we only present the ECG intervals which are defined by FPs of P- and T-waves: $T P_{\text {int }}=T_{\text {peak }}-P_{\text {peak }}, P_{d u r}=P_{o f f}-P_{\text {on }}$ and $T_{d u r}=T_{o f f}-T_{o n}$.

\subsection{Data and Evaluation metrics}

4.4.1. Database for ECG denoising: We use the MIT-BIH arrhythmia database (DB1) (MITBIH-DB1) and MIT-BIH noise stress test database (DB2) (MITBIHDB2). Records of DB1 and DB2 were sampled at $360 \mathrm{~Hz}$. In brief for showing the denoising performance, we use the following records:

- Record 119 of DB1 which has PVC beats

- Records 111, 113, 115, 116, 117, 121, 122, 123, 124 and 231 from DB1 which we will use for providing noisy records by adding Gaussian (white) noise to the original signal

- Records $118 \mathrm{e} 06$ and 118e12 from DB2

4.4.2. Database for ECG fiducial point extraction and interval analysis: We use the Physionet QT database (QT Database, Laguna et al 1997), and a swine ECG database (DB3) (Sayadi et al 2014) that include ECG signal annotations by physicians. QT database contains the annotated ECG records of different databases. Here we use the annotated records of normal sinus rhythm (DB4) and arrhythima database (DB5) in 
the QT databse. Records of the swine ECG database (DB3) were sampled at 1000 Hz. Records of DB4 and DB5 were originally sampled at $250 \mathrm{~Hz}$ and then, resampled to $1000 \mathrm{~Hz}$ to obtain a unified temporal resolution across databases. In brief, we use the following records for ECG FP extraction and interval analysis:

- Records Ischemia01, Ischemia02, Ischemia05, Ischemia09, Ischemia10 and Ischemia 12 (total 1100 beats) of the Swine database (DB3).

- Records Sel16539, Sel16786, Sel16795 and Sel17453 (total 108 beats) of DB4.

- Records Sel100, Sel103, Sel116, Sel117, Sel123, Sel230 and Sel231 (total 197 beats) of DB5.

4.4.3. Evaluation Metrics For quantitative evaluation of a FP extraction method, we calculate estimation error defined as time differences between cardiologist annotations and results of the method. Quantitative results are reported using common metrics: mean (m), standard deviation (s) and root mean square error (RMSE), defined as:

$$
R M S E=\sqrt{M S E}=\sqrt{\frac{1}{N} \sum_{j=1}^{N}\left(e_{j}\right)^{2}}=\sqrt{\left(m^{2}+s^{2}\right)}
$$

where $e_{j}=\hat{y}_{j}-y_{j}$ is denoted as the $j^{\text {th }}$ element of estimation error vector and $N$ is the length of error vector (number of annotations). $y_{j}$ and $\hat{y}_{j}$ are the $j^{\text {th }}$ cardiologist annotation and estimated point, respectively. $m, s$ and $R M S E$ are given in msec. Since the RMSE considers both mean and standard deviation of error, it is a more relevant parameter for comparing the methods.

\section{Results}

\subsection{Results for ECG denoising}

5.1.1. Results for noisy records with additive white noise: By adding Gaussian (white) noise to the original signal with various SNR values, we obtain a noisy signal which we will use to evaluate the efficacy of denoising by the EKF models. Fig.5 shows the denoising results on a part of record 119 of the MIT-BIH arrhythmia database. In this figure we present typical realizations of the original, noisy and estimated (denoised) ECG signals using each method. The subtraction of the original from the estimated ECG signal (error) by each method is also presented on Fig.5, right (note the change of scale). One can observe that both EKF25 models with nonlinear phase exhibit smaller error than the other methods. We find that the nonlinear EKF25 with four observations provides the best result and for input SNR $-4 \mathrm{~dB}$, we obtained 11.8dB of SNR improvment defined as:

$$
S N R_{\text {improved }}[d B]=S N R_{\text {out }}-S N R_{\text {in }}=10 \log \left(\frac{\sum_{i}\left|x_{n}(i)-x(i)\right|^{2}}{\sum_{i}\left|x_{d}(i)-x(i)\right|^{2}}\right)
$$

where, $x$ denotes the original ECG, $x_{d}$ is the denoised signal and $x_{n}$ represents the noisy ECG.

To quantitatively evaluate the performance of the proposed algorithms, we calculate the SNR improvement with respect to the input SNR, using (18). 

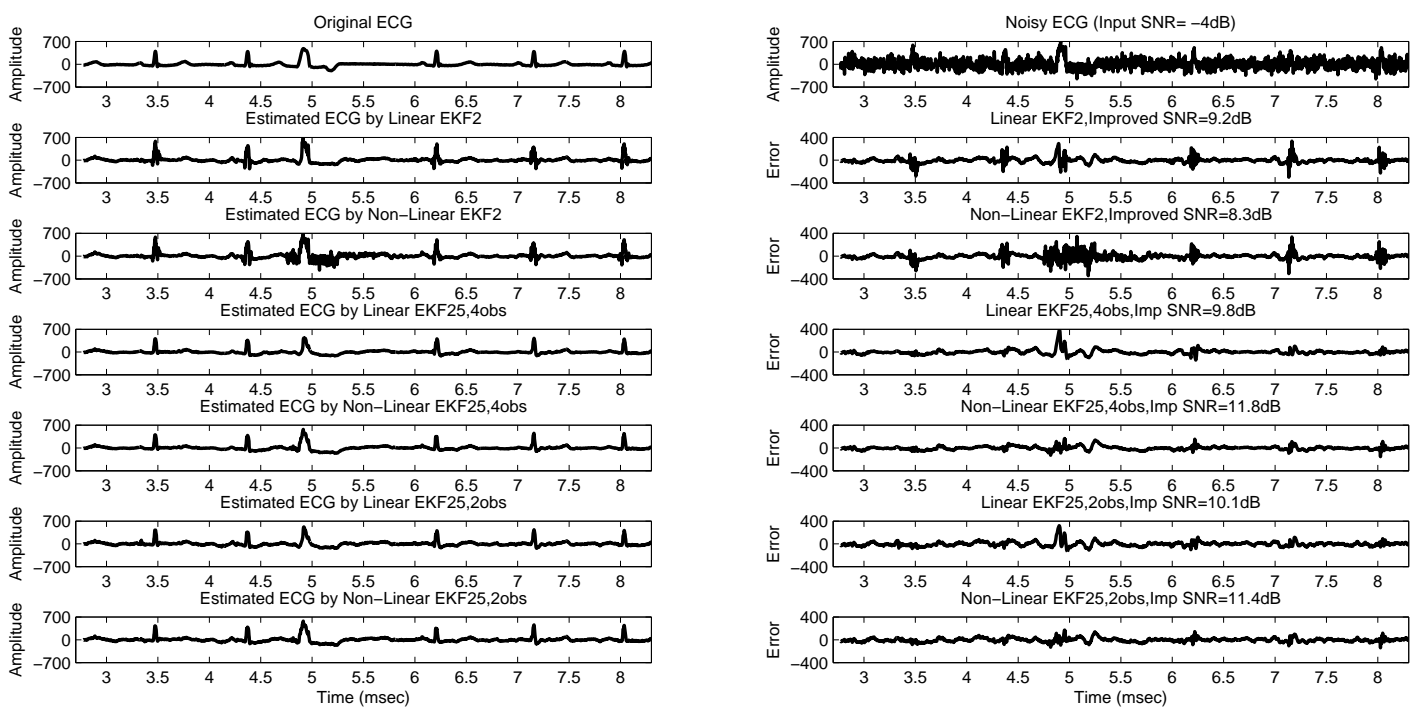

Figure 5. Left, from top to bottom: Original tracing of record 119 of the MITBIH arrhythmia database and estimated ECG signal by (i) linear and nonlinear EKF2, (ii) linear and nonlinear EKF25 with four observations, (iii) linear and nonlinear EKF25 with two observations. Right, from top to bottom: Noisy ECG (input SNR $-4 \mathrm{~dB}$ ), subtraction of the original from the estimated ECG signal by linear and nonlinear EKF2, linear and nonlinear EKF25 with four observations, linear and nonlinear EKF25 with two observations.

To ensure the consistency of the results, the whole procedure was repeated 20 times over the 18 seconds of record no.119, each time using a different set of random white additive noise, as the input. The resulting SNR was averaged across all input SNR values. For a quantitative comparison, in Fig. 6 we plot the mean and standard deviation (SD) of SNR improvement versus different input SNRs for record no. 119.

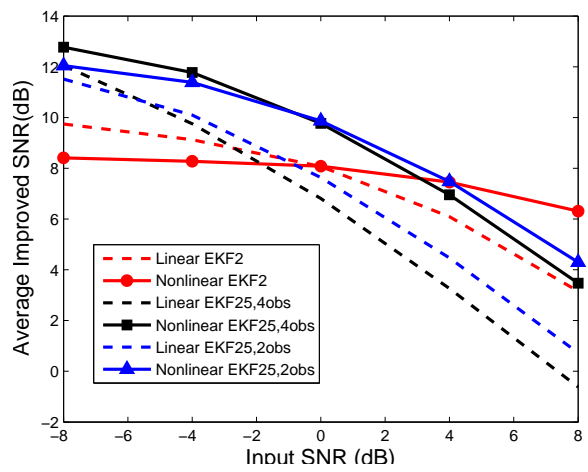

(a)

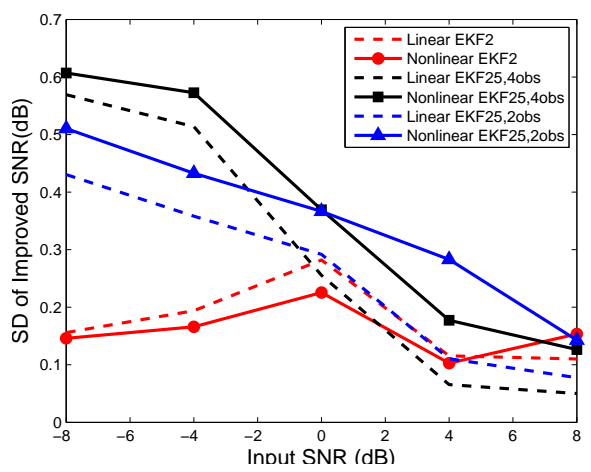

(b)

Figure 6. (a) Mean and (b) SD of the SNR improvement vs. input SNR for record no. 119 of MIT-BIH arrhythmia database.

We also perform the same simulations for 60 seconds of 10 records (records no. 
$111,113,115,116,117,121,122,123,124$ and 231), of the MIT-BIH arrhythmia database. The mean and standard deviation of the SNR improvement versus different input SNRs are plotted in Fig.7.

In Figs.6 and 7, one observes that among all algorithms, (i) the SNR improvement is higher for low input SNR, (ii) the nonlinear EKF25 models perform better than the linear EKF25 models, and (iii) both EKF25-2obs and EKF25-4obs models provide higher SNR improvement compared to EKF2. It is worth to mention that Fig.6 shows the performance of proposed methods for ECG denoising of record 119 which has normal and PVC beats. This figure demonstrates the improvement gained using the non-linear approach. For MIT-BIH arrhythmia database, improvements of the nonlinear approach (Fig.7) are weak, due to the fact that we did not have large RR variations.

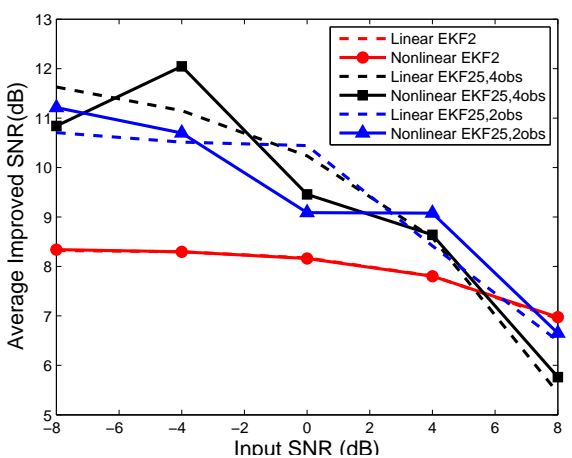

(a)

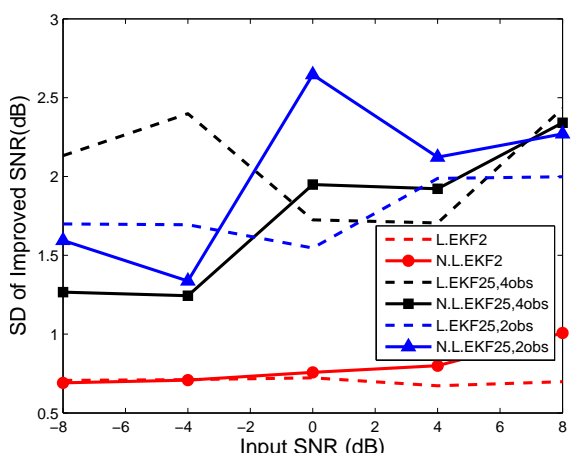

(b)

Figure 7. (a) Mean and (b) SD of the SNR improvement vs. input SNR for 10 records of MIT-BIH arrhythmia database.

We use a paired right-sided Wilcoxon signed rank test (Wilcoxon Signrank Test) to statistically compare all methods, pair by pair. This test shows that EKF25, linear or nonlinear, with 2 or 4 observations, is significantly better than EKF2, linear or nonlinear, with a P-value less than 0.0001 .

5.1.2. Results for noisy records with real noise: Records $118 \mathrm{e} 06$ and $118 \mathrm{e} 12$ from MIT-BIH noise stress test database (DB2) have been contaminated with real electromyogram (EMG) noise and motion artifact. The noisy ECG signal as well as the denoised one using the linear EKF25-2obs and EKF25-4obs models are shown in Fig.8. The signal to noise ratio (SNR) of records $118 \mathrm{e} 06$ and $118 \mathrm{e} 12$ are 6 and $12 \mathrm{~dB}$, respectively. One observes that the denoised signals are free from EMG noise and motion artifact. Since we do not have the original (clean) ECG for these records, so we just show the qualitative results and can not calculate the improved SNR for them. In this figure, our aim is to show the ability of EKF25 models in denoising noisy records with real noise, not comparing the methods.

\subsection{Results for ECG fiducial point extraction}

For ECG FP extraction we only use linear and nonlinear EKF25 with four observations and only compare them with PCGS (presented in 2.2) and EKF17 (presented in 2.1), 


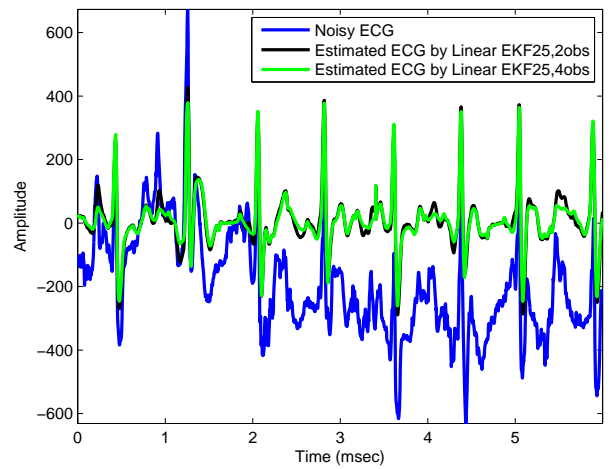

(a)

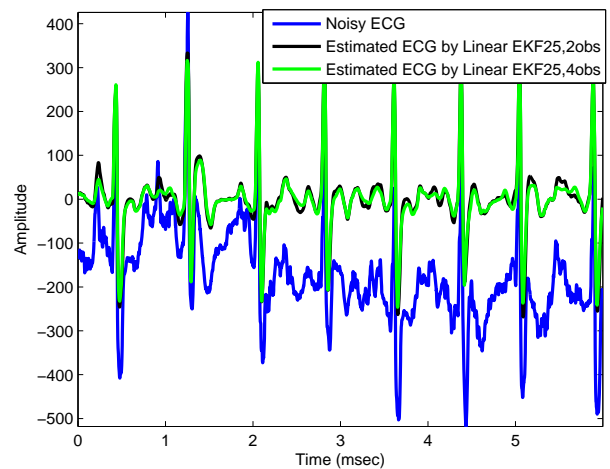

(b)

Figure 8. Noisy ECG and denoised ECG signal using the linear EKF25 methods with two and four observations: (a) record $118 \mathrm{e} 06$ and (b) record 118e12.

since the results of PCGS and EKF17 for FP extraction are superior than other benchmark methods (Lin et al 2010, Sayadi et al 2009).

5.2.1. An example of applying proposed methods: Fig.9 shows the absolute estimation errors of the linear and nonlinear EKF25 for the peak, onset and offset of the P-wave (Fig.9.(b)), of the QRS complex (Fig.9.(c)) and of the T-wave (Fig.9.(d)) of a normal ECG signal (Sel16539). Although this record is a normal ECG signal, it exhibits a very large RR interval variation (as shown in Fig.9.(a)). Therefore, it is not suitable to consider a linear phase observation. We notice that the nonlinear EKF25 provides more accurate FP than the linear EKF25, except for $Q R S_{\text {on }}$. We observe that for FPs such as $P_{\text {peak }}, Q R S_{o f f}, T_{\text {peak }}$ and $T_{o f f}$, the linear EKF25 approach is sensitive to the $\mathrm{RR}$ interval: for large RR interval variations, its estimation error is high, whereas when using the nonlinear EKF25, the estimation error is reduced.

5.2.2. Performance evaluation for each FP in each database: The mean, standard deviation and RMSE of estimation errors of all methods for all databases are presented in tables 2 and 3. RMSE values are presented in parentheses and the best result among all methods are denoted as bold in these tables. In these tables, the algebraic error is calculated. A negative error indicates that the position of the estimated FP occurs before the physician annotation, whereas positive error indicates a late estimation.

Figs.10 and 11 show the distribution of the estimation error of different methods for the onset and offset of P- and T- waves for different databases, respectively. Data are presented as median (horizontal solid line), $25^{t h}$ to $75^{t h}$ percentiles (box) and $10^{t h}$ to $90^{\text {th }}$ percentiles (error bars).

According to tables 2 and 3 and Figs.10 and 11, we observe that for the swine database (DB3) for all FPs except $P_{\text {on }}, P_{\text {peak }}$ and $T_{\text {peak }}$, at least one of the EKF25 models achieves smaller errors than PCGS and EKF17. In addition, for $P_{o f f}, Q R S_{o n}$ and $T_{\text {off }}$, the nonlinear EKF25 provides better results than the linear EKF25.

Estimation of all FPs except $T_{o n}$ and $T_{\text {peak }}$, using the DB4 database indicates that at least one of the EKF25 models achieves smaller errors than PCGS. Moreover, we can remark that for all FPs except $Q R S_{\text {on }}$ and $R_{\text {peak }}$, the nonlinear EKF25 leads 

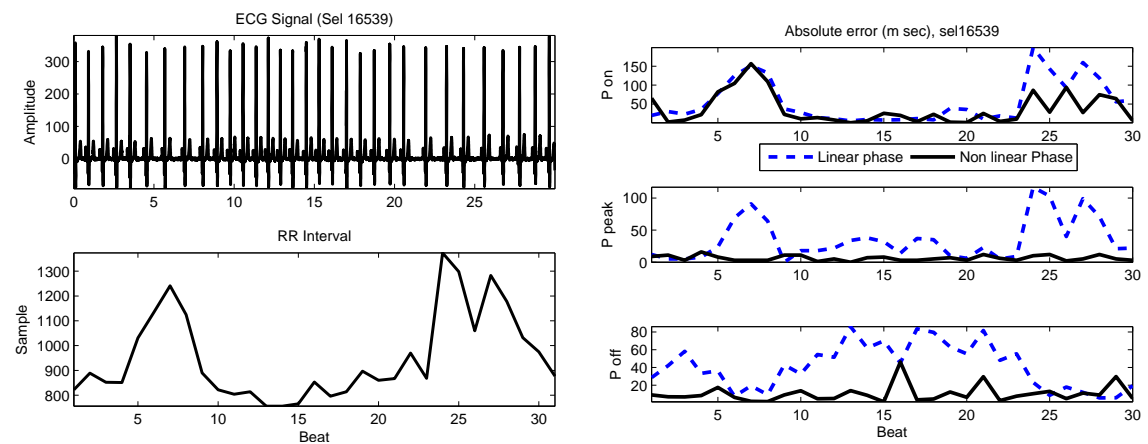

(a)

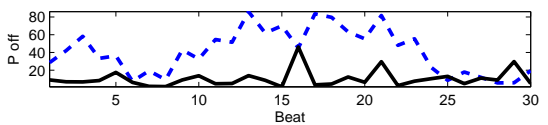

(b)
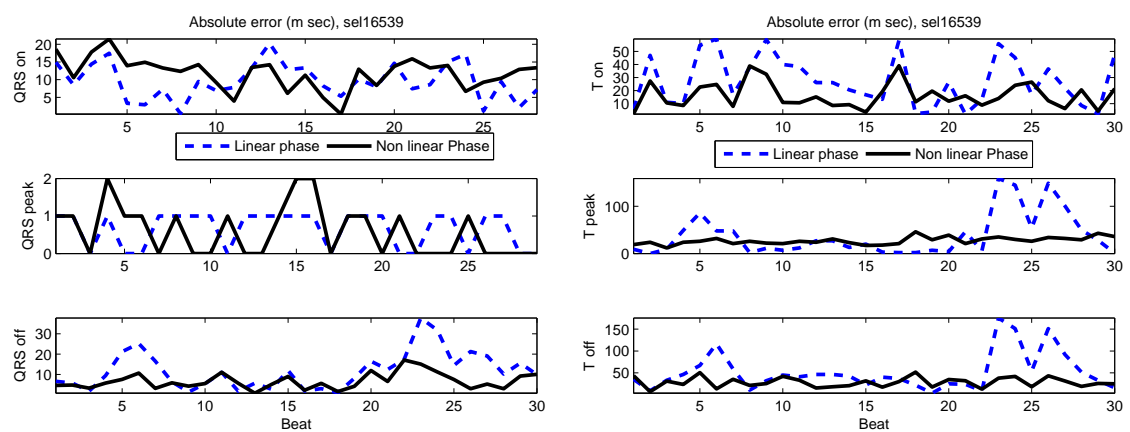

(c)

(d)

Figure 9. Original ECG and its RR interval (a) and the absolute error of Pwave(b), QRS complex (c), and T-wave (d) detection for sel16539 for linear and nonlinear EKF25 with 4 observations. In panels (b)-(d), the absolute errors are shown from top to bottom for the onset, peak and offset, respectively.

Table 2. Mean, standard deviation and RMSE of errors between estimated onset and offset of waves and manual annotations for different databases.

\begin{tabular}{|c|c|c|c|c|c|c|c|}
\hline & & \multicolumn{6}{|c|}{$m \pm s(R M S E) m s e c$} \\
\hline Data & Method & $P_{\text {on }}$ & $P_{o f f}$ & $Q R S_{\text {on }}$ & $Q R S_{o f f}$ & $T_{\text {on }}$ & $T_{o f f}$ \\
\hline \multirow[t]{4}{*}{ DB3 } & L.EKF 25 & $1.2 \pm 25(25)$ & $-3 \pm 12(12.4)$ & $-19 \pm 21(28)$ & $7 \pm 21(\mathbf{2 2})$ & $15 \pm 27(\mathbf{3 1})$ & $-6.5 \pm 11(12.8)$ \\
\hline & N.L.EKF 25 & $0.1 \pm 26(26)$ & $-2 \pm 11(\mathbf{1 1 . 2})$ & $-18 \pm 20(\mathbf{2 7})$ & $8 \pm 22(23)$ & $16 \pm 27(31.4)$ & $-5.5 \pm 11(\mathbf{1 2 . 3})$ \\
\hline & EKF17 & $2.6 \pm 21(21)$ & $8.7 \pm 18(20)$ & $-18 \pm 25(31)$ & $15 \pm 28(32)$ & $17 \pm 38(42)$ & $9 \pm 22(23.8)$ \\
\hline & PCGS & $0.5 \pm 18(\mathbf{1 8})$ & $9.5 \pm 12(15)$ & N.A & N.A & $30 \pm 27(41)$ & $-5.8 \pm 12(13.3)$ \\
\hline \multirow[t]{4}{*}{ DB4 } & L.EKF25 & $-13 \pm 41(43)$ & $19.5 \pm 24(31)$ & $-10 \pm 26(\mathbf{2 8})$ & $10 \pm 15(18)$ & $27 \pm 34(43)$ & $11 \pm 39(41)$ \\
\hline & N.L.EKF 25 & $-7 \pm 36(\mathbf{3 7})$ & $11 \pm 16$ & $-11 \pm 30(32)$ & $8 \pm 15$ & $28 \pm 30(41)$ & $4 \pm 23$ \\
\hline & EKF17 & $-18 \pm 37(41)$ & $26 \pm 27(37)$ & $-15 \pm 35(38)$ & $58 \pm 88(105)$ & $31 \pm 56(64)$ & $20 \pm 42(46)$ \\
\hline & PCGS & $-45 \pm 41(61)$ & $32 \pm 25(41)$ & N.A & N.A & $23 \pm 24(\mathbf{3 3})$ & $25 \pm 17(30)$ \\
\hline \multirow[t]{4}{*}{ DB5 } & L.EKF 25 & $11 \pm 22(\mathbf{2 4})$ & $2 \pm 18(\mathbf{1 8})$ & $-46 \pm 42(62)$ & $11 \pm 35(\mathbf{3 7})$ & $N . A$ & $-17 \pm 30(34)$ \\
\hline & N.L.EKF 25 & $10 \pm 27(29)$ & $6 \pm 16(27)$ & $-43 \pm 42(\mathbf{6 0})$ & $16 \pm 36(40)$ & N.A & $-21 \pm 19(\mathbf{2 8})$ \\
\hline & EKF 17 & $2 \pm 37(37)$ & $22 \pm 24(33)$ & $-57 \pm 64(86)$ & $47 \pm 72(86)$ & N.A & $16 \pm 41(44)$ \\
\hline & PCGS & $-0.4 \pm 38(38)$ & $31 \pm 42(52)$ & $N . A$ & N.A & N.A & $16 \pm 46(49)$ \\
\hline
\end{tabular}

to better performance than the linear EKF25. For all FPs at least one of the EKF25 models achieves smaller errors compared to EKF17.

Estimation of all FPs, using the DB5 database indicates that at least one of the EKF25 models exhibits smaller errors than PCGS and EKF17. In addition, for $P_{\text {peak }}$, $Q R S_{\text {on }}$ and $T_{\text {off }}$, the nonlinear EKF25 appears to be an improved method compared to the linear EKF25. 
Table 3. Mean, standard deviation and RMSE of errors (msec) between estimated peak of waves and manual annotations for different databases.

\begin{tabular}{|l|l|c|c|c|}
\hline & & \multicolumn{3}{|c|}{$m \pm s(R M S E) m s e c$} \\
\hline Data & Method & $P_{\text {peak }}$ & $R_{\text {peak }}$ & $T_{\text {peak }}$ \\
\hline DB3 & L.EKF25 & $0.5 \pm 5.6(5.62)$ & $0.6 \pm 1.4(\mathbf{1 . 5})$ & $-1.2 \pm 5(5.2)$ \\
& N.L.EKF25 & $1.2 \pm 8(8.1)$ & $0.4 \pm 2.6(2.6)$ & $-0.6 \pm 5.6(5.7)$ \\
& EKF17 & $3.3 \pm 4(\mathbf{5 . 2})$ & $1.3 \pm 1.5(2)$ & $-4 \pm 9(9.9)$ \\
& PCGS & $2.6 \pm 6.6(7.1)$ & $N . A$ & $-1 \pm 4(\mathbf{4 . 2})$ \\
\hline DB4 & L.EKF25 & $4 \pm 27(27.3)$ & $-0.3 \pm 1(\mathbf{1 . 0 4})$ & $15 \pm 30(33.5)$ \\
& N.L.EKF25 & $9 \pm 8(\mathbf{1 2 . 1})$ & $-0.6 \pm 1(1.2)$ & $-2 \pm 18(18.2)$ \\
& EKF17 & $4 \pm 28(28.3)$ & $0.7 \pm 2.6(2.7)$ & $12 \pm 41(42.7)$ \\
& PCGS & $12 \pm 18(21.6)$ & $N . A$ & $14 \pm 8(\mathbf{1 6 . 2})$ \\
\hline DB5 & L.EKF25 & $10 \pm 15(18)$ & $0.2 \pm 2(\mathbf{2 . 0 1})$ & $10 \pm 17(\mathbf{1 9 . 7})$ \\
& N.L.EKF25 & $9 \pm 13(\mathbf{1 5 . 8})$ & $1 \pm 3(3.2)$ & $11 \pm 22(24.6)$ \\
& EKF17 & $10 \pm 16(18.9)$ & $-0.5 \pm 9(9.01)$ & $9 \pm 27(28.5)$ \\
& PCGS & $18 \pm 32(36.7)$ & $N . A$ & $21 \pm 32(38.3)$ \\
\hline
\end{tabular}
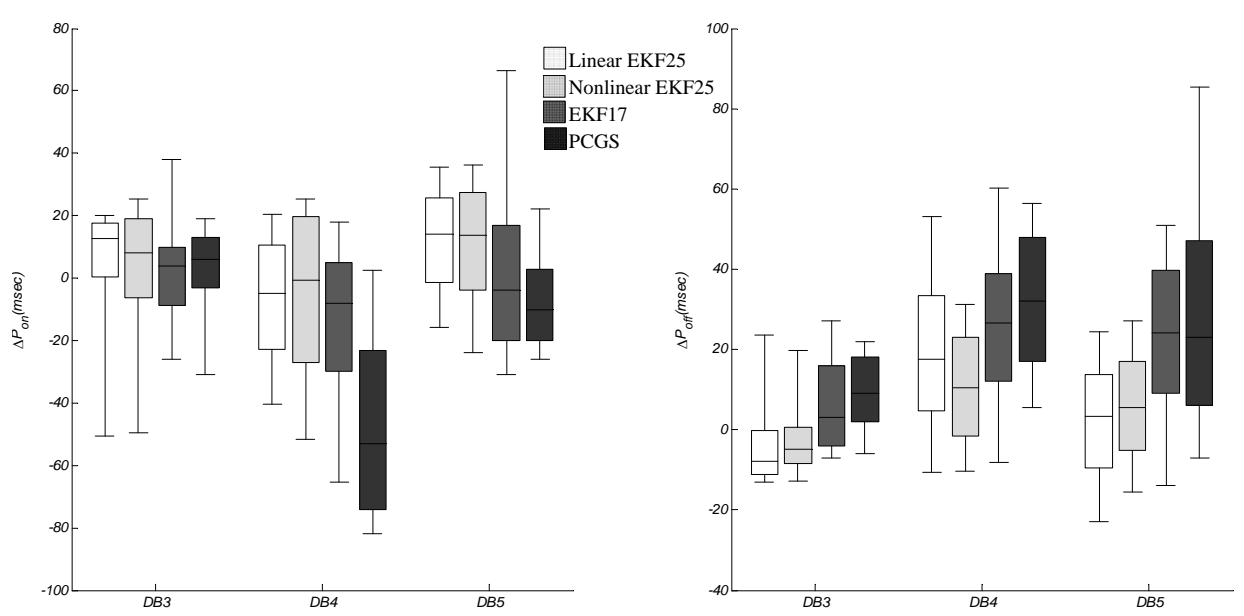

Figure 10. The distribution of estimation error of different methods for $P_{o n}$ (left) and $P_{o f f}$ (right) for different databases.

5.2.3. Performance evaluation for all FPs in each database: In table 4, we present the mean, standard deviation and RMSE of the absolute error among all FPs in each database. Also, the last row of this table presents the absolute error of all FPs, across all databases. In this table, we use the absolute error definition to prevent cancelling out the positive and negative error values of different FPs and different databases.

Table 4. Mean, standard deviation and RMSE of absolute error across all FPs for different databases.

\begin{tabular}{|l|c|c|c|c|}
\hline & \multicolumn{4}{|c|}{$m \pm s(R M S E) m s e c$} \\
\hline Data & $L . E K F 25$ & $N . L . E K F 25$ & $E K F 17$ & $P C G S$ \\
\hline DB3 & $12 \pm 15(\mathbf{1 9 . 2})$ & $12.3 \pm 15(19.4)$ & $14.7 \pm 19.4(24.4)$ & $12.8 \pm 15.6(20.2)$ \\
\hline DB4 & $21.2 \pm 25(32.8)$ & $18 \pm 18.1(\mathbf{2 5 . 5})$ & $32 \pm 43(53.6)$ & $28 \pm 24(36.9)$ \\
\hline DB5 & $20 \pm 23.7(31)$ & $20.3 \pm 23.3(\mathbf{3 0 . 9})$ & $28 \pm 38(47.2)$ & $26 \pm 35(43.6)$ \\
\hline All & $13.78 \pm 17.7(22.4)$ & $13.76 \pm 17(\mathbf{2 1 . 9})$ & $17.7 \pm 25.8(31.3)$ & $15.6 \pm 20.5(25.7)$ \\
\hline
\end{tabular}

We use the Wilcoxon rank-sum test with Bonferroni correction (Wilcoxon 

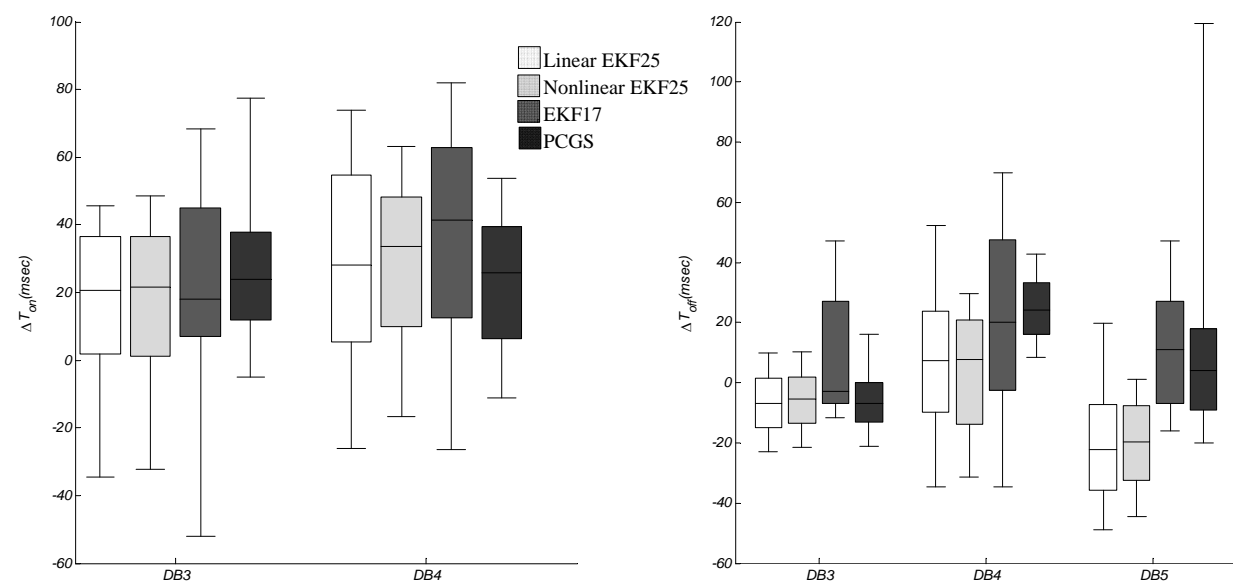

Figure 11. The distribution of estimation error of different methods for $T_{o n}$ (left) and $T_{\text {off }}$ (right) for different databases.

Ranksum Test) to statistically compare all method pairs (linear EKF25, nonlinear EKF25, EKF17 and PCGS). Pairwise comparisons showed a statistically significant difference between any two methods (P-value $<0.0001)$.

In Table 4, we observe that for all three databases and also for aggregate results across all databases, the mean, standard deviation and RMSE of the error for both EKF25 models are smaller than EKF17 and PCGS. In addition, for all databases, the nonlinear EKF25 exhibits similar or superior results than the linear EKF25.

It is worth mentioning that the swine database (DB3) includes ECG signals acquired during acute myocardial infarction, and that exhibit significant morphologic changes (such as ST elevation and QT prolongation) compared to the control signals. Then, we observe that for this database the mean error of the proposed models is smaller than the other methods. The DB4 database includes signals with significant RR interval variability. Then, the proposed methods and especially the nonlinear EKF25 exhibits smaller error than the other ones. Finally, in the DB5 database (which also includes signals with different morphologic abnormalities), the methods presented in this paper can detect FPs more accurately than previously described methods.

To assess the degree of agreement between each of the automated methods and the manual annotations, we used the Bland-Altman approach (Bland et al 2010) to estimate the mean difference and the standard deviation of the difference among all FPs, across all databases. The mean difference and the limits of agreement (defined as twice the standard deviation of differences) were estimated for linear EKF25, nonlinear EKF25, EKF17, and PCGS methods as $-0.44 \pm 44.8,-0.03 \pm 43.6,4.6 \pm 62$ and $7.6 \pm 49.2 \mathrm{msec}$, respectively. The mean differences as well as the margins of agreement for EKF25 annotations are smaller than other methods: it means that they can find fiducial points more accurately than previously described methods. 


\subsection{ECG Interval Analysis}

We calculate the mean, standard deviation and RMSE of the estimation errors obtained using all methods in estimating the ECG intervals. These results are presented in Table 5. Since, in the DB5 database, the physician annotations of the $T_{\text {on }}$ are not available, the results for $T_{\text {on }}$ in table 2 and $T_{d u r}$ in table 5 , are presented as $N$.A.(non available). Fig.13 shows the distribution of estimation error obtained by each method for $P_{d u r}, T P_{\text {int }}$ and $T_{d u r}$ for different databases.

Based on columns 3 to 5 of table 5 and Fig.13, we observe that for the swine database (DB3), (i) estimation of the $T P_{\text {int }}$ and $T_{d u r}$, using both EKF25 models exhibit smaller errors than PCGS, (ii) across all intervals, EKF25 models exhibit smaller errors compared to EKF17. In the DB4 database, estimation of the $P_{d u r}$, using the EKF25 models exhibit smaller errors than the PCGS. It must also be noted that for all intervals, the nonlinear EKF25 achieves better performance than the linear EKF25 and also for $P_{d u r}$ and $T P_{i n t}$, the EKF25 models exhibit smaller errors compared to the EKF17. In the DB5 database estimation of all intervals, using both EKF25 models exhibit smaller errors than PCGS and EKF17.

Table 5. Mean, standard deviation and RMSE of errors (msec) between estimated ECG intervals and manual annotations and differences among all intervals for different databases.

\begin{tabular}{|l|l|c|c|c|c|}
\hline & & \multicolumn{4}{|c|}{$m \pm s(R M S E)$ msec } \\
\hline Data & Method & P duration & TP interval & T duration & All Intervals \\
\hline DB3 & L.EKF25 & $-4 \pm 25(25.3)$ & $-1.8 \pm 7.4(\mathbf{7 . 6})$ & $-21 \pm 30(\mathbf{3 6 . 6})$ & $20 \pm 17.6(\mathbf{2 6 . 6})$ \\
& N.L.EKF25 & $-2.3 \pm 28(28.1)$ & $-2 \pm 9.5(9.7)$ & $-21 \pm 31(37.5)$ & $21 \pm 18.3(27.8)$ \\
& EKF17 & $6 \pm 35(35.5)$ & $-7.5 \pm 11.2(13.5)$ & $-9 \pm 40(41)$ & $24.4 \pm 21.5(32.5)$ \\
& PCGS & $9 \pm 19(\mathbf{2 1})$ & $-4 \pm 7.5(8.5)$ & $-35 \pm 33(48)$ & $20.5 \pm 23(30.8)$ \\
\hline DB4 & L.EKF25 & $32 \pm 40(51.3)$ & $11 \pm 50(51.2)$ & $-16 \pm 43(46)$ & $35 \pm 38(51.7)$ \\
& N.L.EKF25 & $18 \pm 34(\mathbf{3 8 . 5})$ & $-11 \pm 19(22)$ & $-24 \pm 29(37.6)$ & $25 \pm 23(\mathbf{3 4})$ \\
& EKF17 & $44 \pm 33(55)$ & $8 \pm 59(59.5)$ & $-18 \pm 39(43)$ & $40 \pm 36(53.8)$ \\
& PCGS & $76 \pm 43(87.3)$ & $3 \pm 19(\mathbf{1 9 . 3})$ & $3 \pm 32(\mathbf{3 2 . 2})$ & $39 \pm 40(55.8)$ \\
\hline DB5 & L.EKF25 & $-9 \pm 30(\mathbf{3 1 . 3})$ & $0.6 \pm 26(\mathbf{2 6})$ & $N . A$ & $22 \pm 19(\mathbf{2 9 . 1})$ \\
& N.L.EKF25 & $-4 \pm 34(34.3)$ & $3 \pm 29(29.2)$ & $N . A$ & $25 \pm 20.5(32.3)$ \\
& EKF17 & $21 \pm 55(59)$ & $-1 \pm 36(36)$ & $N . A$ & $34.7 \pm 34.8(49.2)$ \\
& PCGS & $31 \pm 38(49)$ & $4 \pm 45(45)$ & $N . A$ & $32 \pm 34(46.7)$ \\
\hline
\end{tabular}

In the last column of table 5, we present the mean, standard deviation and RMSE of absolute error among all intervals in each database.

Here, the mean and standard deviation of absolute error of all intervals, across all databases are estimated for linear EKF25, nonlinear EKF25, EKF17 and PCGS methods as $21.3 \pm 20.5,21.8 \pm 19,26.6 \pm 25$ and $23 \pm 26.7 \mathrm{msec}$, respectively and RMSE values are estimated for above-mentioned methods as 29.6, 28.9, 36.5 and 35.3 msec, respectively. We observe that the mean, standard deviation and RMSE of the absolute error of the EKF25 models are smaller than those of other mentioned methods. Finally, in the DB4 database, the nonlinear EKF25 exhibits much smaller error than the other methods.

We use the Wilcoxon rank-sum test with Bonferroni correction to statistically compare all method pairs (linear EKF25, nonlinear EKF25, EKF17 and PCGS). We observe that all methods are statistically different from each other ( $\mathrm{p}$-value $<0.0001$ ). 


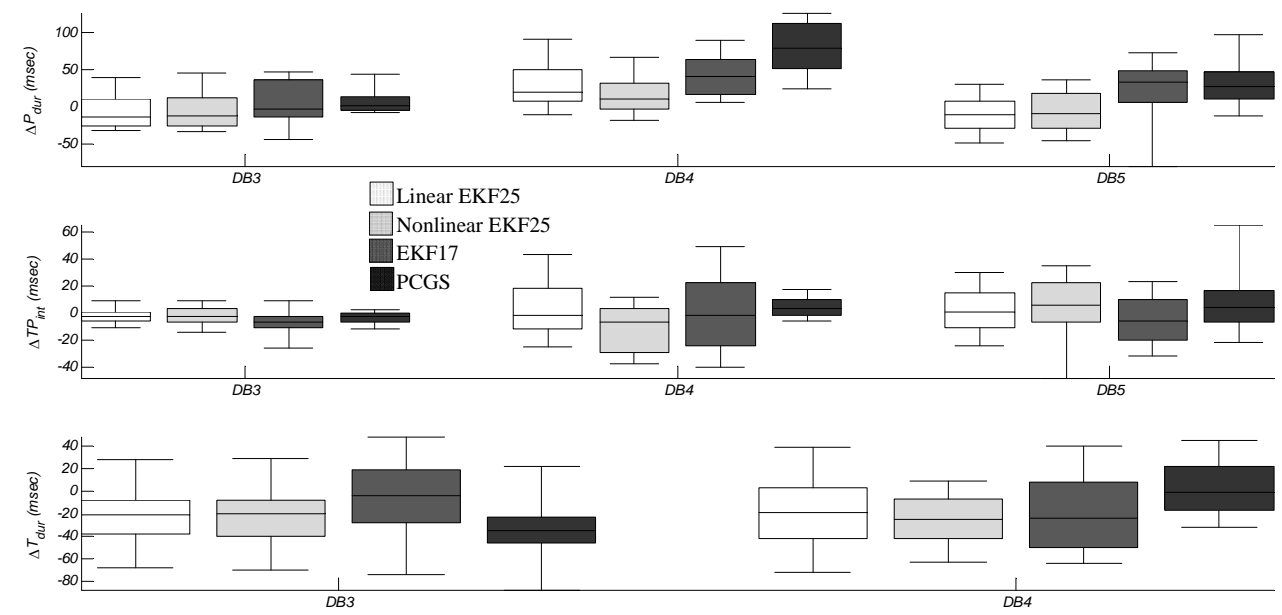

Figure 12. The distribution of estimation error of different methods for $\mathrm{P}$-wave duration (top), TP interval (middle) and T-wave duration (bottom) for different databases new.

\section{Discussion and Conclusions}

In this paper, we propose a method for ECG denoising and fiducial point extraction. The method is based on a nonlinear dynamic model which assumes that each ECG beat is a combination of 7 Gaussian functions with 3 adjustable parameters (amplitude, width and location). By introducing a first-order AR model for each of the 21 dynamic parameters of the Gaussian functions and considering separate states for ECG waveforms (P-wave, QRS complex and T-wave), a new dynamic model with 25 states is constructed, called "EKF25". As this model is nonlinear, an extended Kalman filter is used to estimate the state variables. In EKF25 model, in order to form the observations, we considered two cases: a model with two observations and a model with four observations.

Overall, the advantages of the EKF25 model are: first, the ability to estimate the parameters of Gaussian functions without having any corresponding observations that were used for FPs estimation; second, the ability to estimate separate ECG waveforms, each of which representing a particular physiological state of the heart; third, the ability to find the P- or T-waves of a signal including bi-phasic P-or T-waves, since we model each $\mathrm{P}$ and $\mathrm{T}$ waves with two Gaussian functions.

In this paper, we used the DTW method in order to define a nonlinear phase observation for our proposed model. Use of nonlinear phase observation is suitable for cardiac dysrhythmias such as PVCs, where the nonperiodic abnormal morphology appears only occasionally, and also is suitable for normal ECG signals with large RR-interval variability.

We presented three applications for our proposed model: ECG denoising, fiducial point extraction and interval analysis. For ECG denoising, when applied to ECG signals with frequent PVCs, our method showed a higher SNR improvement than EKF2 especially in lower input SNRs and more importantly in the case of nonlinear phase observation. By applying the nonlinear EKF25 with four observations, a SNR improvement of $12 \mathrm{~dB}$ was achieved for an input SNR of $-8 \mathrm{~dB}$. We also evaluated the 
performance of the above mentioned models in 10 records of the MIT-BIH arrhythmia database and showed that EKF25 models outperform the EKF2 model. We observed that EKF25 models can denoise the ECG signals contaminated with EMG noise and motion artifact.

For ECG fiducial point extraction, we only used the EKF25 model with four observations. We compared the linear and nonlinear EKF25 with PCGS and EKF17 models. Our results showed that the EKF25 method could accurately detect all nine FPs (peak, onset and offset of P-wave, QRS complex and T-wave).

The aggregate results across all databases, indicate that the mean, standard deviation and RMSE of the absolute error for both EKF25 models are smaller than EKF17 and PCGS and the nonlinear EKF25 exhibits better results than the linear EKF25. Both EKF25 models exhibit significantly improved results especially for signals preceding an arrhythmia, during underlying ischemia or signals with large RR-interval variability.

For ECG interval analysis, the mean, standard deviation and RMSE of the absolute error of the EKF25 models are smaller than previously described methods, and the nonlinear EKF25 exhibits similar or superior results than the linear EKF25. When applied to signals with large RR interval variability (DB4 databse), the nonlinear EKF25 exhibited much smaller error than all other methods.

The runtime of the proposed method for a 6 seconds record takes about 1.5 seconds (using a Core i3, 2.53 GHz CPU), suggesting that this method may be used in real-time applications. Investigation and comparison the computational complexity and time for all methods can been done in future work.

The main aim of this study is to improve the detection capacity of previous Kalman filtering frameworks. Advantage of FP estimation based on Kalman filter is that it does not require many parameters to estimate, contrary to non-model-based methods. However, comparison including such models is considering in future works.

Although there are several Bayesian filters such as the Unscented Kalman Filter (UKF), in this work, we have chosen the EKF for its simplicity. The ability of UKF in fiducial point extraction can be examined in the future.

The main limitation of the proposed method is its sensitivity to the initial location of the Gaussian functions as well as initial parameters of EKF, that must be defined by the user. Thus, future works include automatic estimation of these parameters.

\section{Acknowledgment}

This work has been partly supported by the PhD scholarship of French Embassy and the European project ERC-2012-AdG-320684-CHESS.

\section{References}

Afonso V X, Tompkins W J, Nguyen T Q and Luo Sh 1999 ECG beat detection using filter banks IEEE Trans. Biomed. Eng. 46 192-201

Akhbari M, Shamsollahi M B and Jutten C 2012 ECG denoising using angular velocity as a sState and an observation in an extended Kalman filter framework EMBC2012 (USA) 2897-2900

Akhbari M, Shamsollahi M B and Jutten C 2013a Fiducial points extraction and characteristic waves detection in ECG signal using a model-based Bayesian framework ICASSP2013 1257-61

Akhbari M, Shamsollahi M B and Jutten C 2013b ECG Fiducial points extraction by extended Kalman filtering TSP2013 (Rome, Italy) 628-32

Andreao. R, Dorizzi. B and Boudy. J 2006a ECG signal analysis through hidden Markov models IEEE. Trans. Biomed. Eng. 53 1541-9 
Andreao. R and Boudy. J 2006b Combining wavelet transform and hidden Markov models for ECG segmentation EURASIP Journal on Advances in Signal Processing

Ardhapurkar Sh, Manthalkar R and Gajre S 2012 ECG denoising by modeling wavelet subBand coefficients using kernel density estimation J. Inf. Process. Syst. 8 669-84

Bland J M and Altman D G 2010 Statistical methods for assessing agreement between two methods of clinical measurement International Journal of Nursing Studies 47 931-36

Bono V, Mazomenos E B, Chen T, Rosengarten J A, Acharyya A, Maharatna K, Morgan J M and Curzen N 2014 Development of an automated updated Selvester QRS scoring System using SWT-based QRS fractionation detection and classification IEEE Journal of Biomedical and Health Informatics 18 193-204

Bonow R O, Mann D L, Zipes D P and Libby P 2011 Braunwald's Heart Disease :A Textbook of Cardiovascular Medicine Elsevier

Christov I and Simova I 2007 Q-onset and T-end delineation: assessment of the performance of an automated method with the use of a reference database Physiol. Meas. 28 213-21

Coast D A, Stern R M, Cano G G and Briller S A 1990 An approach to cardiac arrhythmia analysis using hidden Markov models IEEE Trans. Biomed. Eng. 37 826-36

Dumont J, Hernandez. A. I and Carrault. G 2010 Improving ECG Beats delineation with an evolutionary optimization process IEEE Trans. Biomed. Eng. 57 607-15

Holsinger W P, Kempner K M and Miller M H 1971 A QRS preprocessor based on digital differentiation

Hu Y H, Tompkins W J, Urrusti J L and Afonso V X 1993 Applications of artificial neural networks for ECG signal detection and classification J. Electrocardiology 26 66-73

Hughes N P, Tarassenko L and Roberts S J 2004a Markov Models for automated ECG interval analysis Proc. Neural Information Processing Systems (NIPS 2004) 611-18

Hughes N P 2006 Probabilistic models for automated ECG interval analysis Ph.D. dissertation, Department of Engineering Science, University of Oxford

Kay S M 1993 Fundamentals of statistical Signal Processing: Estimation Theory Prentice Hall PTR

Kohler B, Hennig C and Orglmeister R 2002 The principles of software QRS detection: reviewing and comparing algorithms for detecting this important ECG waveform IEEE Engineering in Medicine and Biology 42-57

Laguna P, Jane R and Caminal R 1994 Automatic detection of wave boundaries in multi-lead ECG signals: validation with the CSE data-base Computers and Biomedical Research 27 45-60

Laguna P, Mark R G, Goldberg A and Moody G B 1997 A Database for evaluation of algorithms for measurement of QT and other waveform intervals in the ECG IEEE, Computers in Cardiology 24 673-76

Li C, Zheng C and Tai C 1995 Detection of ECG Characteristic points using wavelet transforms IEEE Trans. Biomed. Eng. 42 21-28

Li Q and Clifford G D 2012 Dynamic time warping and machine learning for signal quality assessment of pulsatile signals Physiol. Meas. 33 1491-1501

Lin C, Mailhes C and Tourneret J Y 2010 P- and T-Wave delineation in ECG signals using a Bayesian approach and a partially collapsed Gibbs sampler IEEE Trans. Biomed. Eng. 57 2840-49

Lin C, Kail G, Tourneret J Y, Mailhes C and Hlawatsch F 2011a P and T wave delineation and waveform estimation in ECG signals using a block Gibbs sampler ICASSP 2011 (Prague, Czech Republic) 537-40

Lin C, Bugallo M, Mailhes C and Tourneret J Y 2011b ECG denoising using a dynamical model and a marginalized particle filter IEEE Asilomar Conf. on Signals, Systems and Computers

Maheshwari S, Acharyya A, Puddu P E, Mazomenos E B, Leekha G, Maharatna K and Schiariti M 2013 An automated algorithm for online detection of fragmented QRS and identification of its various morphologies Journal of the Royal Society 10 1-18

Martinez J P, Almeida R, Olmos S, Rocha A P and Laguna P 2004 A wavelet-based ECG delineator: evaluation on standard databases IEEE Trans. Biomed. Eng. 51 570-81

Martinez A, Alcaraz R and Rieta J J 2010 Application of the phasor transform for automatic delineation of single-lead ECG fiducial points Physiol. Meas. 31 1467-85

Mazomenos E B, Biswas D, Acharyya A, Chen T, Maharatna K, Rosengarten J, Morgan J, and Curzen N 2013 A low-complexity ECG feature extraction algorithm for mobile healthcare applications IEEE Journal of Biomedical and Health Informatics 17 459-69

McSharry P E, Clifford G D, Tarassenko L and Smith L A 2003 A dynamic model for generating synthetic electrocardiogram signals IEEE Trans. Biomed. Eng. 50 289-94

Mehta S S and Lingayat N S 2008 Detection of P and T-waves in electrocardiogram WCECS (USA)

Mehta S S, Trivedi C R and Lingayat N S 2009 Identification and delineation of QRS complexes in electrocardiogram using fuzzy C-means Algorithm Journal of Theoretical and Applied 
Information Technology 609-17

MITBIH-DB1: MIT-BIH Arrythmia Database 1980 http://www.physionet.org/physiobank/database/mitdb MITBIH-DB2: MIT-BIH Noise Stress Test 1984 http://physionet.org/physiobank/database/nstdb/

Myers C S and Rabiner L R 1981 A comprative study of several dynamic time warping algorithms for connected word recognition American telephone and telegraph company, the BELL system technical journal 60 1389-409

Niknazar M, Rivet B and Jutten C 2012 Application of dynamic time warping on Kalman filtering framework for abnormal ECG filtering ESANN2012 (Bruges, Belgium) 139-44

QT Database http://www.physionet.org/physiobank/database/qtdb

Raghavendra B S, Bera D, Bopardikar A S and Narayanan R 2011 Cardiac arrhythmia detection using dynamic time warping of ECG beats in E-Healthcare systems WoWMoM 2011 (Italy)

Sameni R, Shamsollahi M B, Jutten C and Clifford G D 2007 Nonlinear Bayesian filtering framework for ECG denoising IEEE Trans. Biomed. Eng. 57 2172-85

Sameni R, Shamsollahi M B and Jutten C 2008 Model-based Bayesian filtering of cardiac contaminants from biomedical recordings Physiol. Meas. 29 595-613

Sayadi O and Shamsollahi M B 2008 ECG denoising and compression using a modified extended Kalman filter structure IEEE Trans. Biomed. Eng. 55 2240-48

Sayadi O and Shamsollahi M B 2009 A model-based Bayesian framework for ECG beat segmentation Physiol. Meas. 30 335-52

Sayadi O, Shamsollahi M B and Clifford G 2010a Robust detection of premature ventricular contractions using a wave-based Bayesian framework IEEE Trans. Biomed. Eng. 57 353-62

Sayadi O, Shamsollahi M B and Clifford G 2010b Synthetic ECG generation and bayesian filtering using a Gaussian wave-based dynamical model Physiol. Meas. 31 1309-29

Sayadi O, Puppala D, Ishaque N, Doddamani R, Merchant F M, Barrett C, Singh J P, Heist E K, Mela T, Martinez J P, Laguna P and Armoundas A A 2014 A novel method to capture the onset of dynamic electrocardiographic ischemic changes and its implications to arrhythmia susceptibility Journal of the American Heart Association (JAHA) 3

Soria E, Sober M, Calpe J, Francisco J, Chorro J and Lopez J 1998 Application of Adaptive Signal Processing for Determining the Limits of $\mathrm{P}$ and $\mathrm{T}$ Waves in an ECG IEEE Trans. Biomed. Eng. 45 1077-80

Sun Y, Chan K L and Krishnan Sh M 2005 Characteristic wave detection in ECG signal using morphological transform BMC Cardiovascular Disorders 5

Wilcoxon Ranksum Test, http://www.mathworks.com/help/stats/ranksum.html

Wilcoxon Signrank Test, http://www.mathworks.com/help/stats/signrank.html

Yu B C, Liu S, Lee M, Chen C Y and Chiang B N 1985 A nonlinear digital filter for cardiac QRS complex detection J. Clin. Eng. 10 193-201

Zifan A, Saberi S, Moradi M H and Towhidkhah F 2006 Automated ECG segmentation using piecewise derivative dynamic time warping International J. Biomed. Sciences 1 181-85 